\title{
NILPOTENT GROUPS ACTING ON ABELIAN GROUPS
}

\author{
CHARLES CASSIDY AND GUY LABERGE
}

(Communicated by Ronald M. Solomon)

\begin{abstract}
In this paper, we study certain properties of the group ring of a nilpotent group which are related to commutativity and conjugation. We establish some relations involving conjugates of the elements of the group ring; these relations are then used to get a better understanding of torsion in abelian-bynilpotent groups; we shall see notably that given any action of a nilpotent group $N$ on an abelian group $A$, then the set of torsion elements of $A$ with respect to the action of $N$ is actually a subgroup of $A$.
\end{abstract}

In nilpotent (or even locally nilpotent) groups, torsion elements always form a subgroup; moreover, a nilpotent group can only have multiple roots $\left(x^{n}=y^{n}\right.$ with $x \neq y$ ) if it has torsion elements. In both cases, the situation is quite different if we drop the nilpotency assumption; one can find in [7] an appropriate setting for the study of these questions.

Groups with unique roots as well as groups in which roots exist for each element have been extensively studied in the literature. Baumslag [1,2] studied extensively those concepts in relation notably with wreath products, hence with group extensions. On the other hand, for any fixed set of prime numbers $\omega$, $\mathrm{Kuz}^{\prime}$ min showed in [6] that any metabelian $U_{\omega}$-group $\left(x^{n}=y^{n}\right.$ with $n \in \omega$ implies $x=y$ ) could be embedded in a metabelian $D_{\omega}$-group $\left(x \mapsto x^{n}\right.$ is a bijection whenever $n \in \omega)$.

Let us denote by $P=P(\omega)$ the set of prime numbers complementary to $\omega$. We were able to obtain a new proof of Kuz'min's result by showing that given any extension $A \mapsto G \rightarrow B$ with $A$ and $B$ abelian and $G$ a $U_{\omega}$-group, it is possible to embed $G$ in the quotient of $A_{P} \mid B_{P}$ (the wreath product of the $P$-localizations of $A$ and $B$ ) by its $\omega$-torsion subgroup (the minimal normal subgroup with a $D_{\omega}$-quotient). We used the idea beautifully developed by Bludov and Medvedev [3] in their completion of ordered metabelian groups as well as results to be found in [1,2]. By trying (unsuccessfully) to adapt the same proof to the abelian-by-nilpotent situation, we obtained the results contained in this paper which, we believe, are interesting by themselves.

Let us first introduce some notation and terminology.

- We denote as usual by $R[B]$ the group ring, where $R$ is any commutative ring with a unity element and $B$ is an arbitrary group.

Received by the editors March 10, 1992.

1991 Mathematics Subject Classification. Primary 20C07, 20F18. 
- We define $n(x) \in \mathbb{Z}[B]$ by

$$
n(x)=\sum_{i=0}^{n-1} x^{i}=1+x+\cdots+x^{n-1} .
$$

- We denote by $S_{\omega}(B)$ the multiplicative subset of $\mathbb{Z}[B]$ generated by those $n(x)$ 's with $n$ an $\omega$-number (all prime factors in $\omega) . S_{\omega}(B)$ thus consists of all finite products $n_{1}\left(x_{1}\right) n_{2}\left(x_{2}\right) \cdots n_{k}\left(x_{k}\right)$ with $n_{1}, n_{2}, \ldots, n_{k}$ being $\omega$-numbers and $x_{1}, x_{2}, \ldots, x_{k} \in B$.

- When the group $B$ is acting on an abelian group $A$, that is, when $A$ is a $\mathbb{Z}[B]$-module, we set $R_{\omega}(A)=\left\{a \in A \mid a^{s}=1, s \in S_{\omega}(B)\right\}$; the elements of $R_{\omega}(A)$ can then be seen [4] as the $\omega$-torsion elements of $A$ with respect to the action of $B$ (or $\mathbb{Z}[B]$ ).

- Since any $\alpha \in R[B]$ can be written uniquely as a finite sum $\alpha=\sum_{i=1}^{k} r_{i} x_{i}$ with all $r_{i}$ 's $\neq 0$ and all $x_{i}$ 's $\in B$ different, it makes sense to define

$$
\operatorname{supp}(\alpha)=\left\{x_{i} \mid i=1,2, \ldots, k, \text { in the above expression }\right\}
$$

and then

$$
\operatorname{Gr}(\alpha)=\langle x \in B \mid x \in \operatorname{supp}(\alpha)\rangle,
$$

the subgroup of $B$ generated by $\operatorname{supp}(\alpha)$; if $\alpha, \beta \in R[B]$, we also define

$$
\operatorname{Gr}(\alpha, \beta)=\langle x \in B| x \in \operatorname{supp}(\alpha) \text { or } x \in \operatorname{supp}(\beta)\rangle \text {. }
$$

- If $\alpha \in R[B]$ and $y_{1}, \ldots, y_{n} \in B$, we define

$$
\alpha^{S\left(y_{1}, \ldots, y_{n}\right)}=\alpha^{y_{1}} \alpha^{y_{2}} \cdots \alpha^{y_{n}}=\left(y_{1}^{-1} \alpha y_{1}\right)\left(y_{2}^{-1} \alpha y_{2}\right) \cdots\left(y_{n}^{-1} \alpha y_{n}\right) ;
$$

note that

$$
\alpha^{S\left(y_{1}, \ldots, y_{n}\right)} \alpha^{S\left(z_{1}, \ldots, z_{m}\right)}=\alpha^{S\left(y_{1}, \ldots, y_{n}, z_{1}, \ldots, z_{m}\right)} ;
$$

however, in general, $\alpha^{S(x, y)} \neq \alpha^{S(y, x)}$ even if $x$ and $y$ commute.

Theorem 1. Let $B$ be a nilpotent group and $R$ a commutative ring with unity element. Take $\alpha, \beta \in R[B]$. If $G r(\alpha)$ is abelian, then there exist $z_{1}, \ldots, z_{k} \in$ $\operatorname{Gr}(\alpha, \beta)$ and $\delta \in R[B]$ satisfying

$$
\beta \alpha^{S\left(z_{1}, \ldots, z_{k}\right)}=\alpha \delta .
$$

Proof. We use an induction on $\operatorname{nil}(\operatorname{Gr}(\alpha, \beta))$, the nilpotency class of $\operatorname{Gr}(\alpha, \beta)$.

If $\operatorname{Gr}(\alpha, \beta)$ is abelian, then $\beta \alpha=\alpha \beta$ and the theorem is then obviously satisfied by taking $k=1, z_{1}=1$, and $\delta=\beta$.

We shall now assume that the result is true whenever $\operatorname{nil}(\operatorname{Gr}(\alpha, \beta))=m$ and consider the case where $\operatorname{nil}(\operatorname{Gr}(\alpha, \beta))=m+1$. This step will be proven by another induction as we shall now see.

Suppose that $\beta=n_{1} y_{1}+\cdots+n_{t} y_{t}$. We certainly have

$$
\beta \alpha^{y_{1}}=\left(n_{1} y_{1}\right) \alpha^{y_{1}}+\left(\sum_{i=2}^{t} n_{i} y_{i}\right) \alpha^{y_{1}}=\alpha\left(n_{1} y_{1}\right)+\left(\beta-n_{1} y_{1}\right) \alpha^{y_{1}} .
$$

Assume that we have found $z_{1}, z_{2}, \ldots, z_{k_{1}} \in \operatorname{Gr}(\alpha, \beta)$ and $\delta_{1} \in R[B]$ such that

$$
\beta \alpha^{S\left(z_{1}, \ldots, z_{k_{1}}\right)}=\alpha \delta_{1}+\left(\beta-\left(n_{1} y_{1}+\cdots+n_{j-1} y_{j-1}\right)\right) \alpha^{S\left(z_{1}, \ldots, z_{k_{1}}\right)}
$$


for some $j, 2 \leq j \leq t$. It suffices to show that we can go one step further and obtain a similar formula for $j+1$; this process will of course lead to the desired result (when $j+1=t$ ).

Set $\alpha^{\star}=\alpha^{y_{j}}$ and $\beta^{\star}=\alpha^{S\left(z_{1}, \ldots, z_{k_{1}}\right)}$. Clearly, $\operatorname{Gr}\left(\alpha^{\star}\right)$ is abelian; on the other hand, $y_{j}, z_{1}, \ldots, z_{k_{1}} \in \operatorname{Gr}(\alpha, \beta)$ and $\operatorname{Gr}\left(\alpha^{\star}, \beta^{\star}\right)$ is generated by elements of the form $x^{y}$ with $x \in \operatorname{Gr}(\alpha)$ and $y \in G r(\alpha, \beta)$; it follows that $\operatorname{nil}\left(G r\left(\alpha^{\star}, \beta^{\star}\right)\right) \leq m$ since $\operatorname{Gr}(\alpha)$ is abelian, $\operatorname{Gr}\left(\alpha^{\star}, \beta^{\star}\right)$ is contained in the product of $\operatorname{Gr}(\alpha)$ by the commutator subgroup of $\operatorname{Gr}(\alpha, \beta)$, and $\operatorname{nil}(\operatorname{Gr}(\alpha, \beta))$ $=m+1$. Applying the induction hypothesis to $\alpha^{\star}$ and $\beta^{\star}$, we find $\omega_{1}, \omega_{2}, \ldots$, $\omega_{k_{2}} \in \operatorname{Gr}\left(\alpha^{\star}, \beta^{\star}\right) \quad(\leq \operatorname{Gr}(\alpha, \beta))$ and $\delta_{2} \in R[B]$ such that

$$
\beta^{\star}\left(\alpha^{\star}\right)^{S\left(\omega_{1}, \ldots, \omega_{k_{2}}\right)}=\alpha^{\star} \delta_{2},
$$

that is,

$$
\alpha^{S\left(z_{1}, \ldots, z_{k_{1}}\right)} \alpha^{y_{j} S\left(\omega_{1}, \ldots, \omega_{k_{2}}\right)}=\alpha^{y_{j}} \delta_{2}
$$

or $\alpha^{S\left(z_{1}, \ldots, z_{k_{1}}, y_{j} \omega_{1}, \ldots, y_{j} \omega_{k_{2}}\right)}=\alpha^{y_{j}} \delta_{2}$.

Combining (1) and (2), we get

$$
\begin{aligned}
\beta \alpha^{S\left(z_{1}, \ldots, z_{k_{1}}, y_{j} \omega_{1}, \ldots, y_{j} \omega_{k_{2}}\right)}=\beta \alpha^{S\left(z_{1}, \ldots, z_{k_{1}}\right)} \alpha^{S\left(y_{j} \omega_{1}, \ldots, y_{j} \omega_{k_{2}}\right)} \\
\quad=\alpha \delta_{1} \alpha^{S\left(y_{j} \omega_{1}, \ldots, y_{j} \omega_{k_{2}}\right)}+\left(\beta-\left(n_{1} y_{1}+\cdots+n_{j-1} y_{j-1}\right)\right) \alpha^{y_{j}} \delta_{2} \\
=\alpha \delta_{1} \alpha^{S\left(y_{j} \omega_{1}, \ldots, y_{j} \omega_{k_{2}}\right)}+n_{j} y_{j} \alpha^{y_{j}} \delta_{2}+\left(\beta-\left(n_{1} y_{1}+\cdots+n_{j} y_{j}\right)\right) \alpha^{y_{j}} \delta_{2} \\
=\alpha\left(\delta_{1} \alpha^{S\left(y_{j} \omega_{1}, \ldots, y_{j} \omega_{k_{2}}\right)}+n_{j} y_{j} \delta_{2}\right)+\left(\beta-\left(n_{1} y_{1}+\cdots+n_{j} y_{j}\right)\right) \alpha^{y_{j}} \delta_{2} .
\end{aligned}
$$

If we set $\delta_{3}=\delta_{1} \alpha^{S\left(y_{j} \omega_{1}, \ldots, y_{j} \omega_{k_{2}}\right)}+n_{j} y_{j} \delta_{2}$ and $z_{k_{1}+i}=y_{j} \omega_{i}\left(1 \leq i \leq k_{2}\right)$, we thus obtain

$$
\beta \alpha^{S\left(z_{1}, \ldots, z_{k_{1}+k_{2}}\right)}=\alpha \delta_{3}+\left(\beta-\left(n_{1} y_{1}+\cdots+n_{j} y_{j}\right)\right) \alpha^{S\left(z_{1}, \ldots, z_{k_{1}+k_{2}}\right)}
$$

which proves the theorem.

Corollary 1. Let $B$ and $R$ be as above and $\alpha, \beta \in R[B]$. Suppose that $\alpha=\alpha_{1} \alpha_{2} \cdots \alpha_{n}$ with the $\operatorname{Gr}\left(\alpha_{i}\right)$ 's abelian $(i=1, \ldots, n)$. Then there exist $z_{1,1}, \ldots, z_{1, k_{1}}, z_{2,1}, \ldots, z_{2, k_{2}}, \ldots, z_{n, k_{n}} \in B$ and $\delta \in R[B]$ such that

$$
\beta \alpha_{1}^{S\left(z_{1,1}, \ldots, z_{1, k_{1}}\right)} \alpha_{2}^{S\left(z_{2,1}, \ldots, z_{2, k_{2}}\right)} \cdots \alpha_{n}^{S\left(z_{n, 1}, \ldots, z_{n, k_{n}}\right)}=\alpha \delta .
$$

Proof. Since $\operatorname{Gr}\left(\alpha_{1}\right)$ is abelian, the theorem shows that we can write

$$
\beta \alpha_{1}^{S\left(z_{1,1}, \ldots, z_{1, k_{1}}\right)}=\alpha_{1} \delta_{1} .
$$

We then have $\delta_{1} \alpha_{2}^{S\left(z_{2,1}, \ldots, z_{2, k_{2}}\right)}=\alpha_{2} \delta_{2}$ by applying the theorem another time since $\operatorname{Gr}\left(\alpha_{2}\right)$ is abelian. After $n$ steps, we obtain the desired result.

This corollary specializes to an interesting result about the factorisation of the elements of $S_{\omega}(B)$ in the integral group ring $\mathbb{Z}[B]$ of the nilpotent group $B$.

Corollary 2. Let $\mathbb{Z}[B]$ be the integral group ring of the nilpotent group $B$. Take $\beta \in \mathbb{Z}[B]$ and $s \in S_{\omega}(B)$. There exist $\tilde{s} \in S_{\omega}(B)$ and $\delta \in \mathbb{Z}[B]$ such that $\beta \tilde{s}=s \delta$. In other terms, $S_{\omega}(B)$ is a right Ore set in the ring $Z[B]$ (see [5, Chapter 9]).

Proof. Every $s \in S_{\omega}(B)$ is a finite product of terms of the form $x(n)=1+$ $x+x^{2}+\cdots+x^{n-1}$. Since $\operatorname{Gr}(x(n))$ is the cyclic group generated by $x \in B$, it is abelian and Corollary 1 applies. 
The following result is now a consequence of [5, Lemma 9.3], but we shall include the proof for completion.

Theorem 2. Let $B$ be a nilpotent group and $A$ a $\mathbb{Z}[B]$-module. Then the set $R_{\omega}(A)=\left\{a \in A \mid a^{s}=1, s \in S_{\omega}(B)\right\}$ of all $\omega$-torsion elements of $A$ with respect to the action of $B$ forms a submodule.

Proof. Let us take $a_{1}, a_{2} \in A$ satisfying $a_{1}^{s_{1}}=1$ and $a_{2}^{s_{2}}=1$ where $s_{1}, s_{2} \in$ $S_{\omega}(B)$. We must show that there exists $s \in S_{\omega}(B)$ such that $\left(a_{1} a_{2}^{-1}\right)^{s}=1$, but by Corollary 2 we know that there exist $\gamma \in S_{\omega}(B)$ and $\delta \in \mathbb{Z}[B]$ such that $s_{2} \gamma=s_{1} \delta$. It thus follows that

$$
\left(a_{1} a_{2}^{-1}\right)^{s_{2} \gamma}=a_{1}^{s_{2} \gamma} \cdot\left(a_{2}^{-1}\right)^{s_{2} \gamma}=a_{1}^{s_{2} \gamma}=a_{1}^{s_{1} \delta}=1 .
$$

Let $A \nrightarrow G \rightarrow B$ be a group extension with $A$ abelian and $B$ an arbitrary $U_{\omega}$-group. The $\omega$-torsion subgroup $T_{\omega}(G)$ is then the union of an ascending family $R_{i}$ of submodules of $A$; namely, set $R_{0}=\{1\}$ and define $R_{i}$ inductively as the submodule of $A$ generated by those $a \in A$ such that $a^{s} \in R_{i-1}$ for some $s \in S_{\omega}(B)$. Now, in the special case when the group $B$ is nilpotent, we have, by Theorem 2 , the following result:

Corollary 3. $T_{\omega}(G)=R_{1}=R_{\omega}(A)$.

\section{BIBLIOGRAPHY}

1. G. Baumslag, Wreath products and p-groups, Proc. Cambridge Philos. Soc. 55 (1959), 224231.

2. __ Roots and wreath products, Proc. Cambridge Philos. Soc. 56 (1960), 109-117.

3. V. V. Bludov and N. Ya. Medvedev, On the completion of ordered metabelian groups, Algebra i Logika 13 (1974), 369-373.

4. C. Casacuberta, Espais topològics amb accions P-locals, Ph.D. Thesis, Universitat Autònoma de Barcelona, 1988.

5. K. R. Goodearl and R. B. Warfield, An introduction of noncommutative Noetherian rings, London Math. Soc. Stud. Texts, vol. 16, Cambridge Univ. Press, Cambridge and New York, 1989.

6. Ju. V. Kuz'min, The variety of metabelian D-groups, Math. USSR-Izv. 6 (1972), no. 4, 759-781.

7. P. Ribenboim, Equations in groups, with special emphasis on localization and torsion. I, Atti Accad. Naz. Lincei Mem. Cl. Sci. Fis. Mat. Natur. Ser. Ia (8) 19 (1987), no. 2, 23-60; II, Portugal. Math. 44 (1987), 417-445.

Departement de Mathematiques et de Statistique, Universite laval, Quebec, Canada E-mail address: cassidy@mat.ulaval.ca 